\title{
Are implicitly activated associates selectively activated?
}

\author{
DOUGLAS L. NELSON \\ University of South Florida, Tampa, Florida \\ VANESA M. MCKINNEY \\ State University of New York, Fredonia, New York \\ and \\ CATHY L. MCEVOY \\ University of South Florida, Tampa, Florida
}

\begin{abstract}
What constitutes a word's associative past? Words differ in how many associates they activate in memory and, following a brief encounter, those with fewer associates are more likely to be recalled in the presence of related cues. The issue addressed in the present article is whether associative set size effects are produced through the selective activation of strong associates or through the activation of both strong and weak associates. The set size of the strongest associates was varied factorially with the set size of the associates of these associates. We assume that associate set size indexes a word's weaker associates. Experiments 1 and 2 showed that recall varied inversely with both target and associate set sizes. Such results held over variations in study time and participant age. Experiment 3 showed that weak associates of the target had a greater effect on recall when there were more connections among the strongest associates in the set. The findings suggest that activation is not strength selective but includes both weak and strong associates.
\end{abstract}

Encountering a familiar word appears to activate its related associates implicitly; for example, studying APPLE activates red, orange, fruit, pie, and so on (Underwood, 1965). Such associates play major roles in the construction of meaning during comprehension (Kintsch, 1988), in false memory (Roediger \& McDermott, 1995; Underwood, 1965), in cued recall (Nelson, Schreiber, \& McEvoy, 1992; Nelson \& Zhang, 2000), and in recognition (Nelson, McKinney, Gee, \& Janczura, 1998; Nelson, Zhang, \& McKinney, 2001). In false memory, associates are sometimes mistakenly recalled or recognized as the study word. In recognition and cued recall, memory performance is better when there are more connections among the associates of studied words, or what we call high connectivity. In cued recall, memory also benefits when the test cue used to prompt recall shares associates and mediators with the studied word (target). For shared associates, both the test cue and the target produce the same word as an associate; for example, the cue STONE and the target PEBBLE each produce rock as an associate. For mediators, the cue produces an associate that in turn produces the target: STONE produces rock, which produces PEBBLE.

This research was supported by Grants MH16360 from the National Institute of Mental Health to D.L.N. and AG13973 from the National Institute on Aging to C.L.M. Correspondence concerning this article should be addressed to D. L. Nelson, Department of Psychology, University of South Florida, Tampa, FL 33620-8200 (e-mail: dnelson2@ chuma1.cas.usf.edu).
Associates of the studied target can also interfere with its recall. For example, associates that are not linked directly or indirectly to the test cue disrupt recall. Target words having larger sets of associates are not as likely to be recalled, and this phenomenon is called the target set size effect. Up to this point, however, we do not know whether this interference is produced by only the strongest nonlinking associates or by both the strongest and weakest nonlinking associates. There is evidence that weak associates are less likely than strong associates to intrude as false memories (McEvoy, Nelson, \& Komatsu, 1999), and that activation produced by subthreshold primes does not produce contextual recognition effects, compared with that produced by suprathreshold primes (Balota, 1983). When a word is comprehended and encoded for later recall, only the strongest associates may be selectively activated, or all may be activated, including the very weakest. The question is, what constitutes the associative past of a word when it is encountered out of context?

Research on words with ambiguous meanings (such as BANK) suggests that access may be exhaustive under conditions of low contextual constraint and become more selective as these constraints increase (see Twilley \& Dixon, 2000 , for a recent review). In the extralist cued recall paradigm, in which encoding occurs under minimal contextual constraint, Gee (1997) has shown that associates related to both meanings of ambiguous words interfere during testing. In this paradigm participants study individual words in the absence of related context cues. Recall 
is tested by presenting a cue word that is associatively related to the target and that is unavailable during the study experience. However, this experiment, like the previous cuing experiments mentioned above, measured only the strongest associates of the to-be-remembered target words. Left unanswered is whether the influence on recall extends to weaker associates.

Single-response free association norms were used in these studies to index the number and relative strengths of a word's associates (Nelson, McEvoy, \& Schreiber, 1999). Participants read a word and produced the first related word to come to mind. Strength of association for each associate is determined by counting the percentage of participants who produce it. Set size is determined by counting the number of unique associates in the set. Both indices are highly reliable, and each index is a strong predictor of successful recall (Nelson \& Zhang, 2000). A recent study, however, has shown that the single associate procedure provides a stable indicator of only the strongest associates, defined as those produced by two or more participants (Nelson, McEvoy, \& Dennis, 2000). Set size grew when subjects were asked to free-associate two responses. The newly added associates tended to be very weak, produced by an average of only $1 \%$ of the subjects, and the great majority of second responses had already been produced as first responses by other participants.

Given the results of the dual-response procedure, we cannot determine whether the interference caused by target set size in the earlier studies was produced by the selective activation of the strongest associates or by the exhaustive activation of strong and weak associates. A demonstration showing that weak associates also disrupt cued recall would resolve this ambiguity. Such an experiment requires indices of strong and weak associates, which ideally could be achieved by renorming thousands of words with dual-response procedures. Given that it took 20 years to develop single-response norms for over 5,000 words (Nelson et al., 1999), we decided against this alternative. Instead, we opted for a procedure that relied on existing free association data. If the single-response procedure yields a reliable index of the strongest associates, we assumed that the associates of these associates could capture many of the weakest. In the present experiments, target set size was manipulated as usual by selecting targets that had either small or large sets. This manipulation was crossed with the set size of the target's associates by counting the number of associates linked to each associate in the set and then averaging those values. For example, with APPLE, the associate red has a set size of 14 , orange has a set size of 14 , fruit a set size of 17 , and so on, and these set size values were summed and divided by the target's set size on the basis of single responses. With this procedure words like dessert, which is not produced as a direct associate, are included in the set as weak associates because this word is produced by pie, which is one of APPLE's associates.

Not all of the associates of the associates are likely to be associates of the target, because some could be simply unrelated. A study of the associates of 24 words (List 1) by two judges working independently indicated that about half of the associates of the associates could be reasonably classified as weak associates of the target. For example, APPLE has 17 associates according to the single-associate procedure, and these 17 associates produced another 231 associates that were not associates of APPLE in the original procedure. One hundred four of these associates were judged as being weak associates of APPLE and 127 were judged as unrelated. For example, APPLE produces worm, which produces crawl, and crawl is unlikely to be a weak associate of APPLE. Thus the measure of weak associates used in this study was calculated as one half the total number of associates of the target's associates. This value was then divided by target set size to estimate the average number of weak associates contributed to the target's set by each of its associates, an index that we call associate set size.

The aim of Experiment 1 was to determine whether weakly activated associates would influence recall in the same way as would strongly activated associates. Strongly activated associates were determined using the target set size index and weakly activated associates were measured using the associate set size index. In the extralist cuing procedure, participants studied targets that varied in factorial combinations of target and associate set size and were then shown related words as cues used to prompt recall. Targets with larger sets were not expected to be recalled as well as those with smaller sets, and if weak associates are accessed, associate set size will have similar effects. Recall probability should be lowest when both target and associate set size are large, and highest when both are small. Presentation time during study was varied in Experiment 1 to determine whether associate set size effects would be more apparent at longer presentations that allow more time for processing. Prior work indicates that target set size effects linked to strong associates are independent of study time (e.g., Nelson et al., 1992), and if weak associates are similarly activated, then similar results should be obtained. Experiment 2 replicated this study with younger and older participants. Experiment 3 evaluated the expectation that preexisting connections among the strongest associates will increase the negative effects of weak associates on recall if they are actually members of the target's set.

\section{EXPERIMENT 1}

\section{Method}

Design and participants. The experimental design formed a $3 \times$ $2 \times 2$ factorial, with rate of presentation during study $(1.5,3.0$, and $6.0 \mathrm{sec}$ ) varied between subjects, and target set size (small, large) and associate set size (small, large) varied within subjects. Twentyfour participants were assigned to each between-subjects condition, with 8 assigned to each of 3 lists of words. The participants were selected from introductory courses in psychology and assigned to condition lists in replication blocks, and they received course credit for voluntary participation.

Materials. The three lists of targets along with their test cues are shown in Appendix A. These materials were constructed from free association norms that now include more than 72,000 pairs and are available on the web (Nelson et al., 1999). Each list was subdivided 
into 6 targets representing the four combinations of target and associate set size. When target set sizes were small and large, the sets averaged $6.63(S D=1.34)$ and $19.52(S D=2.58)$ relatively strong associates, respectively. When associate set sizes were small and large, the respective set sizes were $11.48(S D=0.83)$ and $17.74(S D=$ $0.62)$. Some of these associates were also strong associates of the target (e.g., PEBBLE produces rock as a direct associate). When these associates were subtracted, the corrected means were $9.96(S D=1.09)$ and $16.35(S D=0.86)$ for small and large associate set sizes, respectively. To put these values in perspective, targets in the smallsmall condition had an average of 6.63 strong associates and roughly 33 weak associates $(6.63 \times 9.96 / 2)$, on the assumption that approximately half of the associates of the associates were weakly related to the target. Those in the large-large conditions had an average of 19.52 strong associates and roughly 159 weak associates $(19.52 \times$ $16.35 / 2)$. Values for the mixed set size conditions fell in between.

Features known to affect cued recall were controlled within each set size condition. Test cues had weak to moderate forward connections with their targets, averaging $.13(S D=.08)$. Shared associate and mediated strengths averaged $.06(S D=.10)$ and $.02(S D=.03)$, respectively. Cue set size averaged 14.22 associates $(S D=5.34)$. Means for target frequency, concreteness, and number of connections among the associates were $112(S D=239), 4.68(S D=1.42)$, and $1.45(S D=0.47)$. Frequency refers to the number of times per million words that a specific item appears in printed text, and concreteness refers to ratings on a 1-7 scale of how strongly a word's meaning refers to an object or something that can be directly experienced by the senses. The only exception to this pattern of equating values within each set size condition involved backward targetto-cue strength, which averaged 0.16 overall. Because of a few exceptionally strong items, backward strength was unintentionally confounded with target set size so that it was higher $(0.29, S D=$ $0.29)$ when target set size was small than when it was large $(0.04$, $S D=0.05$ ). This confounding was discovered after the experiments were completed, but other than exaggerating the magnitude of the effects of target set size, we thought that it would not pose a problem for interpreting the effects of associate set size. ${ }^{1}$ Neither backward strength nor any of the other variables was confounded with associate set size.

Procedure. Extralist cuing procedures were used. Participants were shown the target words one at a time on a computer screen for $1.5,3.0$, or $6.0 \mathrm{sec}$; they read the words aloud when shown, and they were asked to remember as many words as possible, without being told how they would be tested. Immediately following the last study word, the instructions for the cued recall task were read to them, including an example of how the test words and studied target words were related. The test trial was self-paced, and the order of appearance of all words was independently randomized for each participant.

\section{Results}

As is shown in Table 1, recall was better when target set size was smaller, when associate set size was smaller, and when presentation time was longer. The results of an analysis of variance indicated that each effect was significant. Probability of correct recall was higher when target set size was small (.63) than when it was large (.38) $\left[F(1,69)=216.25, M S_{\mathrm{e}}=.021\right]$. Recall was also higher when associate set size was small (.55) than when it was large $(.45)\left[F(1,69)=41.14, M S_{\mathrm{e}}=0.017\right]$, and although this effect was somewhat more apparent at the longest presentation time, the interaction with presentation time was not reliable $(F=2.24)$. Finally, recall was more likely when items were presented for $6 \mathrm{sec}(.55)$ or for $3 \mathrm{sec}(.54)$ than when they were shown for $1.5 \mathrm{sec}(.42)[F(2,69)=$ $\left.8.86, M S_{\mathrm{e}}=0.058\right]$. Fisher's two-tailed LSD for this comparison was .07. All of the remaining interaction sources produced $F \mathrm{~s}<1$.

\section{EXPERIMENT 2}

Experiment 2 partially replicated the initial study with younger and older participants. Older individuals show target set size effects (McEvoy, Holley, \& Nelson, 1995), and our interest focused on whether they would show associate set size effects as well. Target set size effects are reduced for older adults, presumably because advancing age reduces access to what has been implicitly activated (McEvoy, 2001), and we expected similar patterns of results for both target and associate set size in this experiment.

\section{Method}

Design and Participants. The design was a $2 \times 2 \times 2$ mixedmodel factorial with age (younger, older) varied between subjects, and both target and associate set size varied within subjects. Younger participants were drawn from the same sources as in Experiment 1 (average age 21 years, $S D=2.26$ ), and older participants comprised community volunteers (average age 84 years, $S D=5.25$ ). Both groups were assigned to conditions in replication blocks as before, with 12 assigned to List 1 and 12 to List 2 .

Materials and Procedure. The lists and procedures for Experiment 1 were utilized in this experiment with two exceptions: To reduce the confound of backward strength and target set size, only Lists 1 and 2 were used, and only the 6-sec presentation time was used.

\section{Results}

The findings reported in Table 2 show that probability of correct recall was higher for younger participants, for targets with smaller sets, and for associates with smaller sets. Probability of recall was .58 and .47 for younger and older participants, respectively $\left[F(1,46)=9.97, M S_{\mathrm{e}}=\right.$ 0.059 ], it was .65 and .40 when targets had small and large sets, respectively $\left[F(1,46)=205.03, M S_{\mathrm{e}}=0.016\right]$, and it was .57 and .48 when associate set size was small and large, respectively $\left[F(1,46)=12.43, M S_{\mathrm{e}}=0.025\right]$. The age $\times$ target set size interaction was also significant $[F(1,46)=$

Table 1

Probability of Correct Recall as a Function of Target Set Size, Associate Size, and Presentation Time (Experiment 1)

\begin{tabular}{cccccc}
\hline & \multicolumn{3}{c}{ Set Size of Target and Set Size of Target's Associates } \\
\cline { 2 - 3 } \cline { 5 - 6 } Presentation & \multicolumn{2}{c}{ Small Target } & & \multicolumn{2}{c}{ Large Target } \\
\cline { 2 - 3 } \cline { 5 - 6 } Time & Small Associate & Large Associate & & Small Associate & Large Associate \\
\hline $1.5 \mathrm{sec}$ & .58 & .49 & & .32 & .28 \\
$3.0 \mathrm{sec}$ & .70 & .60 & & .47 & .39 \\
$6.0 \mathrm{sec}$ & .76 & .63 & & .47 & .33 \\
\hline
\end{tabular}


Table 2

Probability of Correct Recall as a Function of Target Set Size, Associate Set Size, and Age of Participants (Experiment 2)

\begin{tabular}{cccccc}
\hline & \multicolumn{3}{c}{ Set Size of Target and Set Size of Target's Associates } \\
\cline { 2 - 3 } \cline { 5 - 6 } Age of & \multicolumn{2}{c}{ Small Target } & & \multicolumn{2}{c}{ Large Target } \\
\cline { 2 - 3 } \cline { 5 - 6 } Participants & Small Associate & Large Associate & & Small Associate & Large Associate \\
\hline Young & .78 & .69 & & .48 & .35 \\
Old & .60 & .53 & & .40 & .36 \\
\hline
\end{tabular}

19.28]. For younger individuals, the probabilities of recalling words with small and large sets were, respectively, .74 and .42 . For older participants, these values were .57 and .40. Although target set size effects were apparent for both younger and older adults (LSD $=.05)$, they were reduced for older adults. A similar but nonsignificant trend was evident for associate set size $(F=1.90, \mathrm{LSD}=.06)$. Younger participants produced probabilities for small and large sets of .63 and .52, respectively, whereas older adults produced probabilities of .50 and .45 . Older adults show both target and associate set size effects, but their target set size effects are significantly reduced in comparison with younger participants and their associate set size effects show a numerical trend in the same direction.

\section{EXPERIMENT 3}

The results of Experiments 1 and 2 suggest that both strong and weak associates of the target affect the extralist cued recall of both younger and older adults. In the absence of biasing context cues, word comprehension does not appear to be selective in the sense of activating only strong associates. Comprehension depends on the activation of weak as well as strong associates, and the activation of both types of associates influences the likelihood of recall. The purpose of Experiment 3 was to determine whether a manipulation linked to the strongest associates would influence the effects of the weaker associates. Previous findings had shown that greater numbers of preexisting connections among the strongest associates of a target word result in higher cued recall (see Nelson \& Zhang, 2000). Theoretically, such connectivity increases the activation levels of both the target and its associates (Nelson et al., 1998; Nelson \& Zhang, 2000). If the weaker associates of the target are activated along with the stronger associates, then the presence of many connections among the strongest associates will increase the influence of the weaker associates. Higher levels of connectivity will increase the magnitude of the associate set size effect. This expectation depends on the assumption that high connectivity among the strongest associates extends directly to the weakest associates.

\section{Method}

Design and Participants. The design was a $2 \times 2$ repeated measures factorial with associate set size (small, large) and target connectivity (high, low) varied within subjects. There were 30 young participants, with 15 assigned to each of 2 lists.

Materials. The two lists of 24 cue-target pairs are presented in Appendix B. All four conditions of associate set size and target con- nectivity were represented in each list. Associate set size corrected for direct associates averaged $10.62(S D=1.71)$ and $16.46(S D=$ 1.25 ), respectively, for small and large sets. Connectivity was estimated by calculating the number of associate-to-associate connections and then dividing by the number of associates in the set. For example, BABY has 19 strong associates and a total of 39 connections among them, so each associate of this word is connected to an average of 2.05 other associates in the set. High- and low-connectivity targets averaged $2.36(S D=0.30)$ and $0.67(S D=0.18)$ connections per associate.

The test cues were controlled on forward $(0.17, S D=0.10)$ and backward $(0.19, S D=0.21)$ strength and on shared associate $(0.04$, $S D=0.07)$ and mediated $(0.02, S D=0.03)$ strength as well. Cue set size $(13.25, S D=4.63)$ and target set size $(12.31, S D=5.04)$ were also controlled, as were target frequency (76 per million, $S D=158$ ) and target concreteness $(5.12, S D=1.32)$.

Procedure. The procedures were the same as those used in Experiment 1 , except that only the 3 -sec presentation time was used.

\section{Results}

The results are shown in Table 3 , and as can be seen, recall was more likely when associate set size was small (.66) than when it was large $(.57)\left[F(1,29)=5.56, M S_{\mathrm{e}}=\right.$ $0.04]$, and when connectivity among the strongest associates was high $(.67)$ than when it was low $(.55)[F(1,29)=$ $\left.38.01, M S_{\mathrm{e}}=0.01\right]$. More importantly, associate set size effects were more apparent when there were many connections among the associates of the target than when there were few $\left[F(1,29)=5.69, M S_{\mathrm{e}}=0.03\right]$. An LSD of .08 indicated that the effects of associate set size were significant only when many connections were present. Greater numbers of connections among the target's strongest associates increase the activation of its weak associates and they are more likely to interfere with target recall.

\section{GENERAL DISCUSSION}

When words are encountered in the context of reading or conversation, they must be comprehended. Comprehension requires the memory system to retrieve related experiences from the past, and this process incorporates the activation of related associates. Prior research on the effects

Table 3

Probability of Correct Recall as a Function of Connectivity and Associate Set Size (Experiment 3)

\begin{tabular}{ccccc}
\hline $\begin{array}{c}\text { Henall Associate } \\
\text { Set Size }\end{array}$ & $\begin{array}{c}\text { Large Associate } \\
\text { Set Size }\end{array}$ & & $\begin{array}{c}\text { Small Associate } \\
\text { Set Size }\end{array}$ & $\begin{array}{c}\text { Large Associate } \\
\text { Set Size }\end{array}$ \\
\hline .75 & .59 & & .56 & .54 \\
\hline
\end{tabular}


of target set size and connectivity indicates that these associates influence the probability that the recently encountered word will be correctly recalled and recognized. This research relies on a laboratory task in which words are briefly studied in the absence of a biasing context, with retention tested by presenting a related word as a test cue (cued recall) or the target itself (recognition). This research, however, has manipulated only the strongest of these associates. The purpose of the present study was to determine whether weakly activated associates influence recall. Set size of the studied target word and set size of the target's associates were factorially varied in the present experiments as indices of the numbers of strong and weak associates, respectively. The findings indicated that both target and associate set size influenced the probability of cued recall. Words having greater numbers of strong competing associates were less likely to be recalled than those with fewer strong associates, and this interference was also apparent for its weaker competing associates. These effects were apparent for younger and older adults, and they did not vary appreciably with how long the targets were studied.

These findings suggest that directly related associates, both strong and weak, are automatically activated whenever a familiar word is encountered, and that such activation creates an implicit representation that can serve as one basis for supporting later recall (see Nelson et al., 1998, and Nelson \& Zhang, 2000, for a general model called PIER 2). This principle applies to both the target and the test cue. The target activates its associates and the cue activates its associates, and cued recall is described as a computation of the intersection of what the target has activated and what is currently activated by the test cue. The activation of preexisting links that bind the cue and the target include forward, backward, shared associate, and mediated connections, and such connections facilitate recall. In contrast, the activation of associates that are unique to the cue or that are unique to the target interferes with recall. Target recovery is seen as following a ratio rule with the positive effects of linking connections reduced by the negative effects of unique associates. The more unique associates there are in the set, the lower the likelihood of recall. In this approach, recall based on the activation of related associates is seen as the net result of both facilitating and interfering effects. Recall may fail in the presence of a very useful cue simply because a competing associate has been activated.

In the context provided by these assumptions, the present findings show that this competition arises as the result of activating weak as well as strong associates of the target. Implicitly activated associates are not selectively activated. Access to related associates appears to be exhaustive under conditions of low contextual constraint (see, e.g., Twilley \& Dixon, 2000). Except when associates are exceptionally strong (e.g., Nelson \& McEvoy, 2002), participants are unaware of their activation and are assumed to spend additional study time on rehearsal, developing an explicit representation of the study word that includes connections to context and to other studied words. They do not appear to spend any time intentionally strengthening preexisting connections of the studied word to its associates, and as a consequence the influence of both strong and weak associates is unchanged by increased study time.

In Experiment 2 older adults showed both target and associate set size effects, but the magnitude of the target set size effect was significantly reduced relative to that for younger adults. Associate set size effects showed a similar but nonsignificant trend. The model attributes the trends toward reduced set size effects shown by older adults to decreased access to the implicit representation of the target (McEvoy, 2001). Prior research has shown that both target set size and target connectivity effects can be substantially reduced for younger adults when attention to the memory task is disrupted by their having to solve multiplication problems just before testing (Nelson et al., 1998). Such results suggest that information that is automatically activated during comprehension is vulnerable to attention switches, and that the age deficits linked to target set size effects are related to reduced abilities to maintain the focus of attention on the memory task.

Experiment 3 showed that associate set size effects are enhanced by the presence of more interconnections among the target's associates. Greater numbers of connections among the target's strongest associates increased the negative influence of its weaker associates. Preexisting connections among the associates of the target increase its cued recall (see, e.g., Nelson et al., 1998) and show priming effects (Nelson \& Goodmon, 2002). Theoretically, associative connectivity strengthens the implicit representation of the target and makes it more accessible to cues that normally produce it as a response (see Nelson \& Zhang, 2000 , for a more formal description). The results of this experiment suggest that associative connectivity among the strongest associates of an encoded target also primes weaker associates within the set. Some of these weaker associates are unique to the target and provide additional competitors for its recall.

Taken together, these experiments suggest that what is activated by a studied word influences its later recall, and that this activation appears to be exhaustive, encompassing weak as well as strong associates. Even weakly related associates can influence recall, either positively if they link the target to its cue or negatively if they are nonlinking competitors of the target. Furthermore, these influences of weaker associates appear to be magnified when there are many as opposed to few connections among the stronger associates constituting the set. The processes of comprehending and encoding a word when it is encountered in text or normal discourse appear to include the construction of its meaning (Kintsch, 1988), and this construction utilizes weak as well as strong associates.

By way of final comment, we note that the present findings regarding associate set size effects can be interpreted as reflecting the effects of direct but weak associates of the target as opposed to the influence of activation spreading from the test cue to the target, to the target's associates, and then to the associates of these associates. The results of 
examining the associates of the associates and the results of manipulating connectivity convinced us that a compelling case for such spread could not be made. If these weak associates were not part of the target's set, then connectivity among its strongest associates would not have differentially affected the magnitude of the associate set size effect. Moreover, recent studies of our association norms by Steyvers and Tenenbaum (2002) indicate that associations conform to a "small world" network (Watts \& Strogatz, 1998). Very short paths between the items characterize such networks, and in our association network only an average of three links is needed to move from any one of the 5,000 words in the norms to any other. Words in memory are richly interconnected, and therefore activation spreading beyond one or two links should quickly overwhelm any memory advantages that might accrue to words with smaller sets of associates (cf. Nelson, Bennett, \& Leibert, 1997).

\section{REFERENCES}

Balota, D. A. (1983). Automatic semantic activation and episodic memory encoding. Journal of Verbal Learning \& Verbal Behavior, 22, 88-104.

GEE, N. R. (1997). Implicit memory and word ambiguity. Journal of Memory \& Language, 36, 253-275.

Kintsch, W. (1988). The role of knowledge in discourse comprehension: A construction-integration model. Psychological Review, 95, 163-182.

McEvoy, C. L. (2001, March). Aging and remembering: How does what you know influence memory for what is new? Paper presented at Southeastern Workers in Memory, Atlanta.

McEvoy, C. L., Holley, P. E., \& Nelson, D. L. (1995). Age effects in cued recall: Forces from implicit and explicit memory. Psychology \& Aging, 10, 314-324.

McEvoy, C. L., Nelson, D. L., \& Komatsu, T. (1999). What is the connection between true and false memories? The differential roles of interitem associations in recall and recognition. Journal of Experimental Psychology: Learning, Memory, \& Cognition, 25, 1177-1194.

Nelson, D. L., Bennett, D. J., \& Leibert, T. W. (1997). One step is not enough: Making better use of association norms to predict cued recall. Memory \& Cognition, 25, 785-796.
Nelson, D. L., \& Goodmon, L. B. (2002). Experiencing a word can prime its accessibility and its associative connections to related words. Memory \& Cognition, 30, 380-398.

Nelson, D. L., \& McEvoy, C. L. (2002). How can the same type of prior knowledge both help and hinder recall? Journal of Memory \& Language, 46, 652-663.

Nelson, D. L., McEvoy, C. L., \& Dennis, S. (2000). What is free association and what does it measure? Memory \& Cognition, 28, 887899.

Nelson, D. L., McEvoy, C. L., \& Schreiber, T. A. (1999). The University of South Florida word association, rhyme, and fragment norms. http://w3.usf.edu/FreeAssociation/

Nelson, D. L., McKinney, V. M., Gee, N. R, \& Janczura, G. A. (1998). Interpreting the influence of implicitly activated memories on recall and recognition. Psychological Review, 3, 299-324.

Nelson, D. L., Schreiber, T. A., \& McEvoy, C. L. (1992). Processing implicit and explicit representations. Psychological Review, 99, 322348.

Nelson, D. L., \& Z ZANG, N. (2000). The ties that bind what is known to the recall of what is new. Psychonomic Bulletin \& Review, 7, 604-617.

Nelson, D. L., Zhang, N., \& McKinney, V. M. (2001). The ties that bind what is known to the recognition of what is new. Journal of Experimental Psychology: Learning, Memory, \& Cognition, 27, 1147-1150.

Roediger, H. L., III, \& McDermott, K. B. (1995). Creating false memories: Remembering words not presented in lists. Journal of Experimental Psychology: Learning, Memory, \& Cognition, 21, 803-814.

Steyvers, M., \& Tenenbaum, J. (2002). Small worlds in semantic networks. Manuscript submitted for publication.

Twilley, L. C., \& Dixon, P. (2000). Meaning resolution processes for words: A parallel independent model. Psychonomic Bulletin \& Review, 7, 49-82.

UNDERWOOD, B. J. (1965). False recognition produced by implicit verbal responses. Journal of Experimental Psychology, 70, 122-129.

Watts, D. J., \& Strogatz, S. H. (1998). Collective dynamics of "small world" networks. Nature, 393, 440-442.

\section{NOTE}

1. Nelson and Zhang (2000) report the results of a multiple regression analysis of 29 extralist cued-recall experiments, including Experiment 1. This analysis statistically controls for correlations between variables, and when associate set size was introduced into the equation for the purposes of this paper, it was a significant predictor of recall along with backward strength, target set size, and other predictors used in the main analysis reported by Nelson and Zhang.

APPENDIX A

Lists used in Experiments 1 and 2

\begin{tabular}{|c|c|c|c|c|c|}
\hline \multicolumn{2}{|c|}{ List 1} & \multicolumn{2}{|c|}{ List 2} & \multicolumn{2}{|c|}{ List 3} \\
\hline Test Cue & Target & Test Cue & Target & Test Cue & Target \\
\hline \multicolumn{6}{|c|}{ Small Target-Small Associate } \\
\hline ALGEBRA & CALCULUS & CRY & SAD & BUCKET & PAIL \\
\hline LAUGH & HAPPY & FINAL & SON & CRY & SOB \\
\hline PROHIBIT & STOP & FATHER & LAST & FEMALE & WOMAN \\
\hline SEASONING & PEPPER & MOMENT & MINUTE & HEEL & TOE \\
\hline STONE & PEBBLE & SMELL & ODOR & LIGHT & BULB \\
\hline TWILIGHT & EVENING & WORK & JOB & WINE & DINE \\
\hline \multicolumn{6}{|c|}{ Small Target_-Large Associate } \\
\hline BEAR & CUB & DOCTOR & SURGEON & CLOSET & HANGER \\
\hline BOAT & SAIL & LAKE & POND & DISAPPEAR & VANISH \\
\hline DOOR & KNOB & SALMON & TUNA & FIX & REPAIR \\
\hline GAS & FUEL & SHAKE & RATTLE & LACK & NEED \\
\hline OAK & ELM & SHOVEL & DIG & QUIET & NOISY \\
\hline SHOVEL & DIG & THIMBLE & THREAD & STUDENT & PUPIL \\
\hline
\end{tabular}


APPENDIX A (Continued)

\begin{tabular}{|c|c|c|c|c|c|}
\hline \multicolumn{2}{|c|}{ List 1} & \multicolumn{2}{|c|}{ List 2} & \multicolumn{2}{|c|}{ List 3} \\
\hline Test Cue & Target & Test Cue & Target & Test Cue & Target \\
\hline \multicolumn{6}{|c|}{ Large Target-Small Associate } \\
\hline ALLY & FRIEND & CAGE & BIRD & ELEPHANT & GRAY \\
\hline BIRTH & BABY & HATE & LIKE & EMERALD & DIAMOND \\
\hline CROWN & ROYAL & LUMBER & TREE & ENJOY & LIKE \\
\hline GRAVITY & FALL & MAGNITUDE & GREAT & FAMILY & FRIEND \\
\hline MEADOW & FLOWER & MAP & DIRECTION & LOG & TREE \\
\hline TICKET & STUB & WEIGHT & LIFT & VIOLET & FLOWER \\
\hline \multicolumn{6}{|c|}{ Large Target-Large Associate } \\
\hline CHAIN & GANG & CELL & BIOLOGY & FABRIC & MATERIAL \\
\hline CLAW & CRAB & GATHERING & PARTY & MEDAL & HONOR \\
\hline FAD & STYLE & MEDAL & HONOR & OPINION & ADVICE \\
\hline NECK & SHOULDER & PROTECT & DEFEND & PLAYGROUND & SWING \\
\hline OVAL & OFFICE & STALK & HUNT & STRUCTURE & FORM \\
\hline PIE & APPLE & SWAY & SWING & WELL-BEING & HEALTH \\
\hline
\end{tabular}

APPENDIX B

Lists used in Experiment 3

\begin{tabular}{|c|c|c|c|}
\hline \multicolumn{2}{|c|}{ List 1} & \multicolumn{2}{|c|}{ List 2} \\
\hline Test Cue & Target & Test Cue & Target \\
\hline \multicolumn{4}{|c|}{ Small Associate_Low Connectivity } \\
\hline COLOR & BLACK & BOXER & FIGHTER \\
\hline COWBOY & INDIAN & CEILING & ROOF \\
\hline HONEY & BEE & ESCALATOR & ELEVATOR \\
\hline SHY & QUIET & EYE & EAR \\
\hline SUCCESS & FAILURE & PENCIL & ERASER \\
\hline TICKET & STUB & SMEAR & SPREAD \\
\hline \multicolumn{4}{|c|}{ Small Associate_-High Connectivity } \\
\hline DESK & CHAIR & ALIKE & SIMILAR \\
\hline ENEMY & FRIEND & FINISH & BEGIN \\
\hline MONK & MONASTERY & JOKE & FUNNY \\
\hline PIPE & CIGAR & RATTLE & BABY \\
\hline SMALL & LARGE & RING & DIAMOND \\
\hline THIRST & HUNGER & SPACE & STAR \\
\hline \multicolumn{4}{|c|}{ Large Associate-Low Connectivity } \\
\hline CLOWN & CIRCUS & CLOSET & HANGER \\
\hline DIP & CHIP & LABEL & TAG \\
\hline DOOR & KNOB & SAFETY & SEATBELT \\
\hline FIX & REPAIR & SLAVERY & BONDAGE \\
\hline WANDER & ROAM & WALNUT & PECAN \\
\hline WHEEL & TIRE & WANT & NEED \\
\hline \multicolumn{4}{|c|}{ Large Associate-High Connectivity } \\
\hline FARM & CROPS & AISLE & CORRIDOR \\
\hline OVEN & BAKE & BOURBON & ALCOHOL \\
\hline RESEARCH & EXPERIMENT & CELL & BIOLOGY \\
\hline SHOVEL & DIG & GORILLA & APE \\
\hline ТООТН & DECAY & PATROL & $\mathrm{COP}$ \\
\hline UKULELE & GUITAR & sow & SEED \\
\hline
\end{tabular}

(Manuscript received July 17, 2001;

revision accepted for publication January 26, 2002.) 